\title{
Social determinants of denture/bridge use: Japan gerontological evaluation study project cross-sectional study in older Japanese
}

\author{
Tatsuo Yamamoto ${ }^{*}$, Katsunori Kondo ${ }^{2,3}$, Jun Aida ${ }^{4}$, Kayo Suzuki ${ }^{5}$, Jimpei Misawa ${ }^{6}$, Miyo Nakade ${ }^{7}$, Shinya Fuchida ${ }^{1}$,
} Yukio Hirata ${ }^{1}$ and for the JAGES group

\begin{abstract}
Background: Studies suggest that using a denture/bridge may prevent disability in older people. However, not all older people with few remaining teeth use a denture/bridge. This cross-sectional study aimed to examine the social determinants which promote denture/bridge use among older Japanese.

Methods: A total of 54,388 (25,630 males and 28,758 females) community-dwelling individuals aged 65 or over, living independently, able to perform daily activities, and with 19 or fewer teeth. The dependent variable was denture/bridge use. Socio-demographics, number of teeth, present illness, social participation, social support, and social networks were used as individual-level independent variables. Data for social capital were aggregated and used as local district ( $n=561$ for males, $n=562$ for females) -level independent variables. Number of dentists working in hospitals/clinics per population and population density were used as municipality $(n=28)$-level independent variables. Three-level multilevel Poisson regression analysis was performed for each sex.

Results: High equivalent income, low number of teeth, present illness, and living in a municipality with high population density were significantly associated with denture/bridge use in both sexes in the fully adjusted models $(p<0.05)$. Denture/bridge use was significantly associated with high educational attainment in males and participating in social groups in females in the fully adjusted model $(p<0.05)$. No significant associations were observed between denture/bridge use and social capital.

Conclusions: Denture/bridge use was significantly associated with high economic status and present illness in both sexes, high educational attainment in males, and participation in social groups in females among community-dwelling older Japanese after adjusting for possible confounders.
\end{abstract}

Keywords: Social determinants, Dental prosthesis, Older people, Cross-sectional study

\section{Background}

Fixed and removable prostheses are most commonly used to replace missing teeth with the aim of improving chewing ability, aesthetics and pronunciation. Recent studies have reported favorable effects of prosthodontic treatment on systemic health [1-4]. An intervention study showed that prosthodontic treatment improved the nutritional status of institutionalized older people [1]. Cohort studies have reported that older people who do not use

\footnotetext{
* Correspondence: yamamoto.tatsuo@kdu.ac.jp

'Department of Dental Sociology, Kanagawa Dental University Graduate School of Dentistry, 82 Inaoka-cho, Yokosuka, Kanagawa 238-8580, Japan Full list of author information is available at the end of the article
}

dentures despite having few remaining teeth show a higher risk of dementia onset [2] and incident falls [3] after adjusting for possible confounders. Moreover, these studies showed no significant difference in dementia onset and incident falls between subjects having few teeth and using dentures and those having 20 or more teeth, suggesting that denture use may reduce the risk of dementia onset and incident falls in subjects having few teeth. Another cohort study showed that the use of dentures is associated with a decreased risk of mortality in edentulous older people [4].

Despite these favorable effects of dentures and bridges, not all persons who lose their teeth use a denture/bridge. 
In a 2011 Japanese national survey, approximately $29 \%$, $19 \%$, and $14 \%$ of the whole population aged $65-69,70-74$, and 75-79 did not use a denture/bridge, respectively [5]. A study from the UK reported that $25 \%$ and $15 \%$ of patients who were provided with partial dentures never or only occasionally wore dentures, respectively [6].

Denture/bridge use reflects access to dental care. Many factors have been reported to directly and indirectly influence utilization of dental services in older people. These factors can be divided into four main categories: socio-demographic factors, ill health-related factors, service-related factors, and attitude or subjective factors [7]. Socio-demographic factors include sex, age, education, and income $[8,9]$. Ill health-related factors include number of teeth, general ill health, and functional limitations $[10,11]$. Service-related factors include accessibility and insurance coverage $[8,9,12]$. Attitude or subjective factors include personal beliefs and satisfaction with dental visits $[13,14]$. These factors may be associated with denture/bridge use.

Little is known about the factors associated with denture/bridge use. A study among older people in a Japanese municipality revealed an association between denture/ bridge use and economic status [15]. An exploratory qualitative interview study from the UK reported differences in the attitudes of dentists and patients to the provision of removable partial dentures [16]. Dentists focused on restoration of physical function of the teeth, whereas patients focused on the social significance of oral rehabilitation.

It has previously been reported that access to medical care may be associated with social relationships and social capital [17]. However, the association between access to dental care and denture/bridge use and social relationships is unknown, although previous studies have suggested an association between dental health and social relationships $[18,19]$. Individuals obtain health information through their connections with others, and it is possible that this may lead to denture/bridge use. Individuals with few teeth may need to wear denture/bridge to improve appearance or speech, which would help them interact in groups.

The purpose of this study was to examine the social determinants which promote denture/bridge use using cross-sectional data from community-dwelling older Japanese people. First, the association of denture/bridge use with known factors associated with access to dental care (socio-demographic factors, ill health-related factors, and service-related factors) was examined. Then, taking into account these factors, the association with denture/ bridge use and social relationship factors was analyzed. In particular, multilevel (first level, individual; second level, local district; and third level, municipality) analysis was used because it is appropriate to assess contextual and individual determinants of health outcomes, which were not assessed in previous studies on factors associated with access to dental care and denture/bridge use [7-16].

\section{Methods}

\section{Study population}

Data from a cross-sectional study, which were collected as part of the Japan Gerontological Evaluation Study (JAGES) Project, were used for this on-going Japanese prospective cohort study. JAGES aims to conduct empirical studies from gerontological and social epidemiological perspectives. The sample was restricted to those who did not already have a physical or cognitive disability, defined by not receiving public long-term care insurance benefits, at baseline. From July 2010 to January 2012, a mail survey was conducted in a random sample of 169,215 community-dwelling individuals aged 65 years or over residing in 31 municipalities in 12 prefectures in Japan. Of the 112,123 respondents (response rate, 66.3\%), 8,502 subjects were excluded due to a lack of age and sex information. Then, 5,851 subjects in three municipalities were excluded due to a lack of information on local district, social support, and/or general trust. After excluding 411 (lack of information of local district) and 4,525 (status of activities of daily living (ADL) was dependent or unknown) subjects, a total of 92,834 subjects aged 65 or older in 562 local districts in 28 municipalities were included in the present study. The JAGES protocol was reviewed and approved by the Ethics Committee on Research of Human Subjects at Nihon Fukushi University.

\section{Outcome variables}

Dental status was assessed using a self-administered questionnaire [20]. Respondents were asked to classify their dental status as having 20 or more, 10-19, $1-9$ or 0 teeth. Data from all subjects, including those having 20 or more teeth, were used when local district-level social capital was calculated. However, subjects having 20 or more teeth were excluded when univariate and multilevel prevalence ratios (PRs) were calculated. Denture/ bridge use was ascertained by asking, "Do you use a denture or bridge?" with possible answers dichotomized into yes and no.

\section{Socio-demographic variables}

Data on socio-demographics (sex, age, marital status, educational attainment, equivalent income) were obtained using a self-administered questionnaire. To adjust household income for household size, equivalent income was calculated by dividing the household income by the square root of the number of household members, and placed into one of seven categories $(<500,000$ yen, 500,000999,999 yen, 1,000,000-1,499,999 yen, 1,500,000-1,999,999 yen, 2,000,000-2,999,999 yen, 3,000,000-3,999,999 yen, and $\geq 4,000,000$ yen). 


\section{III health-related variables}

Number of teeth and present illness were considered as ill health-related variables. Self-reported current medical treatment for cancer, heart disease, stroke, hypertension, diabetes, obesity, hyperlipidemia, osteoporosis, arthritis, trauma, respiratory disease, gastrointestinal disease, liver disease, mental illness, visual/hearing impairment, dysphagia, urinary disease, sleep disorder, or other conditions was used as the variable present illness, dichotomized into yes and no.

\section{Service-related variables}

Data on the number of dentists working in hospitals or clinics were obtained from the Survey of Physicians, Dentists and Pharmacists conducted by the Ministry of Health, Labour and Welfare, Japan in 2010. Data on population in 2010 and area of inhabitable land of each municipality were obtained from the National Population Census Survey conducted by the Ministry of Internal Affairs and Communications, Japan. Number of dentists working in hospitals or clinics per 100,000 people and population density were calculated for each municipality. The number of dentists working in hospitals or clinics per 100,000 people was categorized into four groups (lowest, low middle, high middle, or highest) based on 25th, 50th, and 75th percentiles. Population density was categorized into four groups (metropolitan, urban, semi-urban, or rural-agricultural).

\section{Social relationship variables}

General trust, norms of reciprocity, and attachment to place were assessed by asking "Generally speaking, would you say that most people can be trusted?", "Would you say that most of the time people try to be helpful?", and "Do you feel attached to the area you live?" with possible answers dichotomized into yes and no (including "depends"). For social participation, respondents were asked whether they belonged to industrial and trade associations, volunteer groups, older people's clubs, sports groups or clubs, neighborhood associations or councils, or hobby clubs, with possible answers dichotomized into yes and no. The number of social groups was calculated for each subject.

Emotional and instrumental social support, both received and given, was evaluated by using the following questions: "Do you have someone who listens to your concerns and complaints?" (emotional social support received), "Do you listen to someone's concerns and complaints?" (emotional social support given), "Do you have someone who looks after you when you are sick and have to stay in bed for a few days?" (instrumental social support received), and "Do you look after someone when he/she is sick and stays in bed for a few days?" (instrumental social support given), with possible answers dichotomized into yes and no.

Social network was measured by the question, "How often do you see your friends?" with the following possible answers: "almost every day", "two or three times per week", "once a week", "once or twice per month", "several times per year", or "rarely".

We created local district $(n=561$ for males, $n=562$ for females) -level social capital variables by aggregating the individual-level data on general trust, norms of reciprocity, attachment to place, social support (both emotional and instrumental received and given), number of social groups, and meeting friends (\% of subjects meeting friends at least several times a year). General trust, norms of reciprocity, attachment to place, and social support were categorized as cognitive social capital. Number of social groups and meeting friends were categorized as structural social capital. Local districts were categorized into four groups (lowest, low middle, high middle, or highest) based on 25 th, 50 th, and 75 th percentiles for each variable.

\section{Analysis}

The following analyses were conducted in subjects with 19 or fewer teeth (25,630 males and 28,758 females). First, univariate PRs and 95\% confidence intervals (CIs) were calculated for each independent variable with denture/bridge use as the dependent variable in each sex. Because the percentage of people using a denture/bridge was high (males: 68.1\%, females: 67.6\%), adjusted odds ratio derived from the logistic regression could no longer approximate PR [21]. Therefore, multilevel Poisson regression model with random intercepts and fixed slopes was used separately for males and females to calculate multilevel PRs, taking into account variations in the outcomes between local districts and municipalities using MLwiN 2.28 (Centre for Multilevel Modelling, University of Bristol, Bristol, UK), with denture/bridge use as the dependent variable [22]. In model 1, socio-demographics (age, marital status, educational attainment, and equivalent income), health status (number of teeth and present illness) and municipality-level characteristics (number of dentists working in hospitals or clinics per 100,000 people and population density) were added. In models 2 and 3, number of social groups and frequency of meeting friends, both of which were significantly associated with denture/ bridge use in the previous univariate analysis, were added to model 1, respectively. Moreover, to examine the association between each local district-level social capital variable and denture/bridge use after adjusting for socio-demographics, health status and municipalitylevel characteristics, each local district-level social capital variable was added to model 1 . In the model, corresponding individual level variable was also added to avoid ecological fallacy. 
Table 1 Association between denture/bridge use and individual-level characteristics in males and females

\begin{tabular}{|c|c|c|c|c|c|c|c|c|}
\hline \multirow[b]{3}{*}{ Characteristic } & \multicolumn{4}{|c|}{ Males, $n=25630$} & \multicolumn{4}{|c|}{ Females, $\mathrm{n}=\mathbf{2 8 7 5 8}$} \\
\hline & \multirow[t]{2}{*}{$\mathbf{n}$} & \multirow{2}{*}{$\begin{array}{c}\text { Denture/bridge } \\
\text { users (\%) }\end{array}$} & \multicolumn{2}{|c|}{ Univariate PR } & \multirow[t]{2}{*}{$\mathbf{n}$} & \multirow{2}{*}{$\begin{array}{c}\text { Denture/bridge } \\
\text { users (\%) }\end{array}$} & \multicolumn{2}{|c|}{ Univariate PR } \\
\hline & & & $P R$ & $95 \% \mathrm{Cl}$ & & & PR & $95 \% \mathrm{Cl}$ \\
\hline \multicolumn{9}{|l|}{ Socio-demographics } \\
\hline \multicolumn{9}{|l|}{ Age group (years) } \\
\hline $65-69$ & 6699 & 69.7 & 1.00 & (reference) & 6923 & 68.5 & 1.00 & (reference) \\
\hline $70-74$ & 7081 & 68.1 & 0.98 & $(0.94-1.02)$ & 7916 & 66.2 & 0.97 & $(0.93-1.01)$ \\
\hline $75-79$ & 6118 & 66.5 & 0.95 & $(0.92-0.99)^{a}$ & 6880 & 67.5 & 0.99 & $(0.95-1.03)$ \\
\hline $80-84$ & 3899 & 68.2 & 0.98 & $(0.93-1.03)$ & 4443 & 68.2 & 1.00 & $(0.95-1.04)$ \\
\hline$\geq 85$ & 1833 & 67.9 & 0.97 & $(0.92-1.04)$ & 2596 & 69.3 & 1.01 & $(0.96-1.07)$ \\
\hline \multicolumn{9}{|l|}{ Marital status } \\
\hline Married & 21449 & 68.7 & 1.00 & (reference) & 15547 & 67.3 & 1.00 & (reference) \\
\hline Separated/divorced & 3216 & 65.9 & 0.96 & $(0.92-1.00)$ & 11868 & 68.6 & 1.02 & $(0.99-1.05)$ \\
\hline Never married & 441 & 60.5 & 0.88 & $(0.78-0.99)^{a}$ & 604 & 66.2 & 0.98 & $(0.89-1.09)$ \\
\hline Unknown/missing & 524 & 63.9 & 0.93 & $(0.83-1.04)$ & 739 & 59.8 & 0.89 & $(0.81-0.98)^{a}$ \\
\hline \multicolumn{9}{|c|}{ Educational attainment (years) } \\
\hline$<6$ & 453 & 62.7 & 1.00 & (reference) & 1028 & 66.4 & 1.00 & (reference) \\
\hline $6-9$ & 11161 & 62.6 & 1.00 & $(0.89-1.12)$ & 13582 & 65.2 & 0.98 & $(0.91-1.06)$ \\
\hline $10-12$ & 7877 & 70.7 & 1.13 & $(1.00-1.27)^{\mathrm{a}}$ & 9512 & 70.2 & 1.06 & $(0.98-1.14)$ \\
\hline$\geq 13$ & 5048 & 77.4 & 1.23 & $(1.10-1.39)^{b}$ & 3171 & 70.7 & 1.06 & $(0.98-1.16)$ \\
\hline Missing & 1091 & 66.0 & 1.05 & $(0.92-1.21)$ & 1465 & 67.8 & 1.02 & $(0.93-1.12)$ \\
\hline \multicolumn{9}{|c|}{ Equivalent income (10000 yen) } \\
\hline$<50$ & 739 & 60.1 & 1.00 & (reference) & 1625 & 60.9 & 1.00 & (reference) \\
\hline $50-99$ & 2192 & 59.6 & 0.99 & $(0.89-1.10)$ & 3274 & 65.0 & 1.07 & $(0.99-1.15)$ \\
\hline $100-149$ & 3214 & 62.2 & 1.04 & $(0.93-1.15)$ & 3151 & 66.4 & 1.09 & $(1.01-1.18)^{a}$ \\
\hline $150-200$ & 5245 & 68.5 & 1.14 & $(1.03-1.26)^{b}$ & 4294 & 68.2 & 1.12 & $(1.04-1.20)^{b}$ \\
\hline $200-299$ & 5452 & 71.6 & 1.19 & $(1.08-1.32)^{c}$ & 4768 & 71.5 & 1.18 & $(1.09-1.26)^{c}$ \\
\hline $300-399$ & 3275 & 75.6 & 1.26 & $(1.14-1.39)^{c}$ & 2760 & 74.5 & 1.22 & $(1.13-1.32)^{c}$ \\
\hline$\geq 400$ & 2266 & 75.2 & 1.25 & $(1.13-1.39)^{c}$ & 2226 & 73.1 & 1.20 & $(1.11-1.30)^{c}$ \\
\hline Missing & 3247 & 62.8 & 1.05 & $(0.94-1.16)$ & 6660 & 63.4 & 1.04 & $(0.97-1.12)$ \\
\hline \multicolumn{9}{|l|}{ Health status } \\
\hline \multicolumn{9}{|l|}{ Number of teeth } \\
\hline $10-19$ & 10407 & 64.9 & 1.00 & (reference) & 11129 & 62.1 & 1.00 & (reference) \\
\hline $1-9$ & 9786 & 73.5 & 1.13 & $(1.09-1.17)^{c}$ & 11565 & 73.7 & 1.19 & $(1.15-1.22)^{c}$ \\
\hline 0 & 5437 & 64.5 & 0.99 & $(0.95-1.04)$ & 6064 & 66.4 & 1.07 & $(1.03-1.11)^{c}$ \\
\hline \multicolumn{9}{|l|}{ Present illness } \\
\hline No & 6192 & 65.1 & 1.00 & (reference) & 6214 & 65.0 & 1.00 & (reference) \\
\hline Yes & 17602 & 69.2 & 1.06 & $(1.03-1.10)^{c}$ & 20081 & 68.2 & 1.05 & $(1.01-1.09)^{b}$ \\
\hline Missing & 1836 & 68.4 & 1.05 & $(0.99-1.12)$ & 2463 & 69.7 & 1.07 & $(1.01-1.13)^{\mathrm{a}}$ \\
\hline \multicolumn{9}{|l|}{ Social relationship } \\
\hline \multicolumn{9}{|l|}{ General trust } \\
\hline No & 1075 & 64.6 & 1.00 & (reference) & 1273 & 64.8 & 1.00 & (reference) \\
\hline Yes & 23326 & 68.3 & 1.06 & $(0.98-1.14)$ & 26111 & 67.8 & 1.05 & $(0.98-1.12)$ \\
\hline Missing & 1229 & 68.1 & 1.05 & $(0.95-1.17)$ & 1374 & 66.8 & 1.03 & (0.94-1.13) \\
\hline
\end{tabular}




\begin{tabular}{|c|c|c|c|c|c|c|c|c|}
\hline \multicolumn{9}{|l|}{ Norms of reciprocity } \\
\hline No & 2237 & 66.5 & 1.00 & (reference) & 2517 & 66.9 & 1.00 & (reference) \\
\hline Yes & 22089 & 68.4 & 1.03 & $(0.98-1.08)$ & 24635 & 67.8 & 1.01 & $(0.96-1.07)$ \\
\hline Missing & 1304 & 66.9 & 1.01 & $(0.92-1.09)$ & 1606 & 67.1 & 1.00 & $(0.93-1.08)$ \\
\hline \multicolumn{9}{|l|}{ Attachment to place } \\
\hline No & 1219 & 67.8 & 1.00 & (reference) & 1285 & 65.8 & 1.00 & (reference) \\
\hline Yes & 23754 & 68.2 & 1.01 & $(0.94-1.08)$ & 26631 & 67.7 & 1.03 & $(0.96-1.10)$ \\
\hline Missing & 657 & 67.0 & 0.99 & $(0.88-1.11)$ & 842 & 68.1 & 1.03 & $(0.93-1.15)$ \\
\hline \multicolumn{9}{|c|}{ Emotional social support (received) } \\
\hline No & 1211 & 64.9 & 1.00 & (reference) & 727 & 64.1 & 1.00 & (reference) \\
\hline Yes & 11573 & 66.7 & 1.03 & $(0.96-1.10)$ & 12619 & 65.7 & 1.03 & $(0.93-1.13)$ \\
\hline Missing & 12846 & 69.8 & 1.07 & $(1.00-1.16)$ & 15412 & 69.4 & 1.08 & $(0.99-1.19)$ \\
\hline \multicolumn{9}{|c|}{ Emotional social support (given) } \\
\hline No & 2225 & 65.0 & 1.00 & (reference) & 1112 & 64.2 & 1.00 & (reference) \\
\hline Yes & 13112 & 67.3 & 1.03 & $(0.98-1.09)$ & 12703 & 65.8 & 1.02 & $(0.95-1.11)$ \\
\hline Missing & 10293 & 70.0 & 1.08 & $(1.02-1.14)^{b}$ & 14943 & 69.5 & 1.08 & $(1.00-1.17)^{a}$ \\
\hline \multicolumn{9}{|c|}{ Instrumental social support (received) } \\
\hline No & 1245 & 65.1 & 1.00 & (reference) & 1380 & 63.9 & 1.00 & (reference) \\
\hline Yes & 15949 & 67.7 & 1.04 & $(0.97-1.12)$ & 16461 & 66.9 & 1.05 & $(0.98-1.12)$ \\
\hline Missing & 8436 & 69.3 & 1.07 & $(0.99-1.15)$ & 10917 & 69.3 & 1.08 & $(1.01-1.16)^{\mathrm{a}}$ \\
\hline \multicolumn{9}{|c|}{ Instrumental social support (given) } \\
\hline No & 2929 & 64.8 & 1.00 & (reference) & 3855 & 66.7 & 1.00 & (reference) \\
\hline Yes & 14467 & 68.2 & 1.05 & $(1.00-1.11)^{a}$ & 13287 & 66.6 & 1.00 & $(0.96-1.04)$ \\
\hline Missing & 8234 & 69.1 & 1.07 & $(1.01-1.12)^{a}$ & 11616 & 69.1 & 1.04 & $(0.99-1.08)$ \\
\hline \multicolumn{9}{|l|}{ Number of social groups } \\
\hline 0 & 6074 & 66.4 & 1.00 & (reference) & 6693 & 66.5 & 1.00 & (reference) \\
\hline 1 & 4390 & 68.5 & 1.03 & $(0.99-1.08)$ & 4551 & 69.9 & 1.05 & $(1.01-1.10)^{\mathrm{a}}$ \\
\hline 2 & 3355 & 70.5 & 1.06 & $(1.01-1.12)^{a}$ & 3306 & 70.2 & 1.06 & $(1.00-1.11)^{\mathrm{a}}$ \\
\hline $3-6$ & 5207 & 71.1 & 1.07 & $(1.02-1.12)^{b}$ & 3871 & 70.2 & 1.06 & $(1.01-1.11)^{\mathrm{a}}$ \\
\hline Missing & 6604 & 66.0 & 0.99 & $(0.95-1.04)$ & 10337 & 65.6 & 0.99 & $(0.95-1.03)$ \\
\hline \multicolumn{9}{|c|}{ Frequency of meeting friends } \\
\hline Rarely & 2866 & 64.5 & 1.00 & (reference) & 1891 & 64.5 & 1.00 & (reference) \\
\hline Several times a year & 5163 & 69.5 & 1.08 & $(1.02-1.14)^{b}$ & 3127 & 68.7 & 1.07 & $(0.99-1.14)$ \\
\hline 1 or 2 times/month & 4986 & 69.7 & 1.08 & $(1.02-1.14)^{b}$ & 5225 & 69.1 & 1.07 & $(1.00-1.14)^{a}$ \\
\hline Once/week & 3595 & 69.1 & 1.07 & $(1.01-1.14)^{a}$ & 4965 & 68.2 & 1.06 & $(0.99-1.13)$ \\
\hline 2 or 3 times/week & 4304 & 67.6 & 1.05 & $(0.99-1.11)$ & 6979 & 67.2 & 1.04 & $(0.98-1.11)$ \\
\hline Almost everyday & 2930 & 68.2 & 1.06 & $(0.99-1.13)$ & 4159 & 67.5 & 1.05 & $(0.98-1.12)$ \\
\hline Missing & 1786 & 65.3 & 1.01 & $(0.94-1.09)$ & 2412 & 65.8 & 1.02 & $(0.95-1.10)$ \\
\hline
\end{tabular}

$\mathrm{PR}$, prevalence ratio; $\mathrm{Cl}$, confidence interval.

${ }^{\mathrm{a}}, p<0.05 ;{ }^{\mathrm{b}}, p<0.01 ;{ }^{c}, p<0.001$.

\section{Results}

The percentages of males and females using a denture/ bridge were $68.1 \%$ and $67.6 \%$, respectively. Table 1 shows the PRs (95\% CIs) for denture/bridge use according to individual-level variables. In both sexes, high equivalent income, low number of teeth, present illness, involvement in two or more kinds of social groups, and meeting friends 1-2 times per month were significantly associated with denture/bridge use. Age group, marital status, educational attainment, and instrumental social support given were associated with denture/bridge use in males. 
Table 2 Association between denture/bridge use and local district- and municipality-level characteristics in males and females

\begin{tabular}{|c|c|c|c|c|c|c|c|c|}
\hline \multirow[b]{3}{*}{ Characteristic } & \multicolumn{4}{|c|}{ Males } & \multicolumn{4}{|c|}{ Females } \\
\hline & \multirow[t]{2}{*}{$\mathbf{n}$} & \multirow{2}{*}{$\begin{array}{c}\text { Denture/bridge } \\
\text { users (\%) }\end{array}$} & \multicolumn{2}{|c|}{ Univariate PR } & \multirow[t]{2}{*}{$\mathbf{n}$} & \multirow{2}{*}{$\begin{array}{c}\text { Denture/bridge } \\
\text { users (\%) }\end{array}$} & \multicolumn{2}{|c|}{ Univariate PR } \\
\hline & & & PR & $95 \% \mathrm{Cl}$ & & & PR & $95 \% \mathrm{Cl}$ \\
\hline \multicolumn{9}{|l|}{ Local district-level characteristics } \\
\hline \multicolumn{9}{|l|}{ Cognitive social capital } \\
\hline \multicolumn{9}{|l|}{ General trust (\%) } \\
\hline Lowest (<92.86) & 85 & 68.6 & 1.00 & (reference) & 85 & 67.0 & 1.00 & (reference) \\
\hline Low middle (92.86 - 97.00) & 304 & 67.8 & 0.99 & $(0.93-1.05)$ & 304 & 67.5 & 1.01 & $(0.95-1.07)$ \\
\hline High middle (97.01 - 99.99) & 114 & 69.2 & 1.01 & $(0.94-1.08)$ & 114 & 68.5 & 1.02 & $(0.96-1.09)$ \\
\hline Highest (100.00) & 58 & 69.8 & 1.02 & $(0.91-1.13)$ & 59 & 68.2 & 1.02 & $(0.92-1.13)$ \\
\hline \multicolumn{9}{|l|}{ Norms of reciprocity (\%) } \\
\hline Lowest (<84.91) & 61 & 71.1 & 1.00 & (reference) & 61 & 68.0 & 1.00 & (reference) \\
\hline Low middle (84.91 - 91.17) & 250 & 69.3 & 0.98 & $(0.90-1.06)$ & 250 & 68.8 & 1.01 & $(0.93-1.10)$ \\
\hline High middle (91.18 - 99.99) & 237 & 67.0 & 0.94 & $(0.87-1.02)$ & 234 & 66.7 & 0.98 & $(0.90-1.06)$ \\
\hline Highest (100.00) & 13 & 71.6 & 1.01 & $(0.77-1.32)$ & 14 & 69.7 & 1.02 & $(0.81-1.30)$ \\
\hline \multicolumn{9}{|l|}{ Attachment to place (\%) } \\
\hline Lowest (<91.03) & 62 & 70.4 & 1.00 & (reference) & 63 & 68.3 & 1.00 & (reference) \\
\hline Low middle (91.03 - 97.11) & 379 & 68.4 & 0.97 & $(0.90-1.05)$ & 379 & 67.9 & 0.99 & $(0.92-1.07)$ \\
\hline High middle (97.12 - 99.99) & 84 & 66.4 & 0.94 & $(0.87-1.03)$ & 84 & 66.3 & 0.97 & $(0.89-1.05)$ \\
\hline Highest (100.00) & 36 & 66.7 & 0.95 & $(0.81-1.10)$ & 36 & 70.3 & 1.03 & $(0.89-1.18)$ \\
\hline \multicolumn{9}{|c|}{ Emotional social support (received) (\%) } \\
\hline Lowest (<88.89) & 142 & 71.9 & 1.00 & (reference) & 143 & 71.2 & 1.00 & (reference) \\
\hline Low middle (88.89 - 92.85) & 139 & 69.7 & 0.97 & $(0.92-1.02)$ & 139 & 69.5 & 0.98 & $(0.93-1.03)$ \\
\hline High middle (92.86 - 95.44) & 139 & 67.5 & 0.94 & $(0.89-0.99)^{a}$ & 139 & 67.5 & 0.95 & $(0.90-1.00)^{a}$ \\
\hline Highest ( $\geq 95.45)$ & 138 & 65.7 & 0.91 & $(0.86-0.97)^{b}$ & 138 & 64.3 & 0.90 & $(0.86-0.95)^{c}$ \\
\hline Missing & 3 & 45.6 & 0.63 & $(0.49-0.83)^{c}$ & 3 & 59.6 & 0.84 & $(0.69-1.01)$ \\
\hline \multicolumn{9}{|c|}{ Emotional social support (given) (\%) } \\
\hline Lowest $(<85.71)$ & 129 & 69.4 & 1.00 & (reference) & 130 & 70.4 & 1.00 & (reference) \\
\hline Low middle (85.71 - 89.35) & 151 & 69.0 & 0.99 & $(0.94-1.04)$ & 151 & 67.8 & 0.96 & $(0.92-1.01)$ \\
\hline High middle (89.36 - 92.09) & 140 & 66.9 & 0.96 & $(0.91-1.01)$ & 140 & 66.3 & 0.94 & $(0.90-0.99)^{\mathrm{a}}$ \\
\hline Highest $(\geq 92.10)$ & 141 & 68.7 & 0.99 & $(0.94-1.04)$ & 141 & 68.5 & 0.97 & $(0.92-1.03)$ \\
\hline \multicolumn{9}{|c|}{ Instrumental social support (received) (\%) } \\
\hline Lowest $(<88.89)$ & 148 & 71.2 & 1.00 & (reference) & 148 & 70.4 & 1.00 & (reference) \\
\hline Low middle (88.89 - 92.26) & 133 & 66.8 & 0.94 & $(0.89-0.99)^{a}$ & 133 & 66.7 & 0.95 & $(0.90-1.00)^{\mathrm{a}}$ \\
\hline High middle (92.27 - 94.81) & 140 & 68.4 & 0.96 & $(0.91-1.01)$ & 140 & 67.9 & 0.97 & $(0.92-1.01)$ \\
\hline Highest ( $\geq 94.82)$ & 140 & 67.8 & 0.95 & $(0.90-1.00)$ & 141 & 67.1 & 0.95 & $(0.91-1.00)$ \\
\hline \multicolumn{9}{|c|}{ Instrumental social support (given) (\%) } \\
\hline Lowest $(<77.42)$ & 140 & 67.9 & 1.00 & (reference) & 141 & 69.1 & 1.00 & (reference) \\
\hline Low middle (77.42 - 81.87) & 140 & 67.6 & 1.00 & $(0.95-1.05)$ & 140 & 66.7 & 0.97 & $(0.92-1.01)$ \\
\hline High middle (81.88 - 85.41) & 141 & 67.5 & 0.99 & $(0.95-1.04)$ & 141 & 67.5 & 0.98 & $(0.93-1.02)$ \\
\hline Highest ( $\geq 85.42$ ) & 140 & 70.3 & 1.03 & $(0.98-1.09)$ & 140 & 68.4 & 0.99 & $(0.94-1.04)$ \\
\hline
\end{tabular}


Table 2 Association between denture/bridge use and local district- and municipality-level characteristics in males and females (Continued)

\begin{tabular}{|c|c|c|c|c|c|c|c|c|}
\hline \multicolumn{9}{|l|}{ Structural social capital } \\
\hline \multicolumn{9}{|l|}{ Mean number of social groups } \\
\hline Lowest $(<1.280)$ & 140 & 69.2 & 1.00 & (reference) & 141 & 70.4 & 1.00 & (reference) \\
\hline Low middle (1.280 - 1.483) & 141 & 69.4 & 1.00 & $(0.95-1.06)$ & 141 & 69.2 & 0.98 & $(0.94-1.03)$ \\
\hline High middle (1.484 - 1.687) & 139 & 68.1 & 0.98 & $(0.94-1.03)$ & 139 & 67.7 & 0.96 & $(0.92-1.01)$ \\
\hline Highest ( $\geq 1.688$ ) & 141 & 67.0 & 0.97 & $(0.92-1.02)$ & 141 & 65.6 & 0.93 & $(0.89-0.97)^{\mathrm{b}}$ \\
\hline \multicolumn{9}{|l|}{ Meeting friends (\%) } \\
\hline Lowest (<87.76) & 136 & 69.8 & 1.00 & (reference) & 137 & 70.2 & 1.00 & (reference) \\
\hline Low middle (87.76 - 91.00) & 142 & 68.6 & 0.98 & $(0.93-1.04)$ & 142 & 68.8 & 0.98 & $(0.93-1.03)$ \\
\hline High middle (91.01 - 93.32) & 137 & 68.8 & 0.98 & $(0.93-1.04)$ & 137 & 67.3 & 0.96 & $(0.91-1.01)$ \\
\hline Highest ( $\geq 93.33)$ & 143 & 66.4 & 0.95 & $(0.90-1.00)$ & 143 & 66.4 & 0.95 & $(0.90-1.00)^{a}$ \\
\hline Missing & 3 & 45.6 & 0.65 & $(0.50-0.85)^{\mathrm{b}}$ & 3 & 59.6 & 0.85 & $(0.70-1.03)$ \\
\hline \multicolumn{9}{|l|}{ Municipality-level characteristics } \\
\hline \multicolumn{9}{|c|}{ Number of dentists per 100000 people } \\
\hline Lowest $(<47.29)$ & 7 & 65.3 & 1.00 & (reference) & 7 & 65.7 & 1.00 & (reference) \\
\hline Low middle (47.29 - 53.97) & 7 & 66.4 & 1.02 & $(0.96-1.07)$ & 7 & 67.5 & 1.03 & $(0.98-1.08)$ \\
\hline High middle (53.98 - 59.74) & 7 & 67.7 & 1.04 & $(0.99-1.09)$ & 7 & 67.4 & 1.03 & $(0.98-1.08)$ \\
\hline Highest $(\geq 59.75)$ & 7 & 70.1 & 1.07 & $(1.02-1.13)^{b}$ & 7 & 68.5 & 1.04 & $(1.00-1.09)$ \\
\hline \multicolumn{9}{|l|}{ Population density $\left(/ \mathrm{km}^{2}\right)$} \\
\hline Rural-agricultural $(<1000)$ & 2 & 64.4 & 1.00 & (reference) & 2 & 64.6 & 1.00 & (reference) \\
\hline Semi-urban (1000-1499) & 7 & 68.1 & 1.06 & $(1.02-1.10)^{b}$ & 7 & 68.4 & 1.06 & $(1.02-1.10)^{b}$ \\
\hline Urban (1500-3999) & 6 & 70.6 & 1.10 & $(1.05-1.14)^{c}$ & 6 & 68.5 & 1.06 & $(1.02-1.10)^{b}$ \\
\hline Metropolitan ( $\geq 4000$ ) & 13 & 72.2 & 1.12 & $(1.08-1.17)^{c}$ & 13 & 72.0 & 1.12 & $(1.07-1.16)^{c}$ \\
\hline
\end{tabular}

$\mathrm{PR}$, prevalence ratio; $\mathrm{Cl}$, confidence interval.

$\mathrm{n}$, number of local districts for local district-level characteristics and number of municipalities for municipality-level characteristics.

a, $p<0.05 i^{\text {b }}, p<0.01{ }^{c}{ }^{c}, p<0.001$.

Table 2 shows the PRs (95\% CIs) for denture/bridge use according to local district- and municipality-level variables. Local district-level emotional and instrumental social support received, meeting friends, and municipalitylevel population density were significantly associated with denture/bridge use in both sexes. Municipality-level number of dentists working in hospitals or clinics per population was associated with denture/bridge use in males, and emotional social support given in females.

Table 3 shows the results of multilevel Poisson regression analyses. High educational attainment, high equivalent income, low number of teeth, present illness, and high population density were significantly associated with denture/ bridge use in males $(p<0.05)$. High equivalent income, low number of teeth present, present illness, involvement in one or more social groups, and high population density were significantly associated with denture/bridge use in females $(p<0.05)$. In both the sexes, frequency of meeting friends was not significantly associated with denture/bridge use in model 3. Significance of the variables in model 1 did not change after adding the variable of frequency of meeting friends in both sexes.
All local district-level social capital variables were not associated with denture/bridge use.

\section{Discussion}

The results of the present study showed that factors independently associated with denture/bridge use in both sexes were equivalent income, number of teeth, present illness, and population density, all of which are known to be associated with access to dental care [7]. In particular, individual financial status was strongly associated with denture/bridge use in the present study, which is in agreement with findings of a study conducted in one municipality in Japan [15]. Studies have suggested that low socioeconomic status is one of the barriers to dental attendance and that such barriers appear to have negative effects on oral health [23,24]. It is noteworthy that even in people with universal free access to dental services under the national healthcare insurance system in Japan, financial issues are a major factor affecting denture/bridge use.

Subjects presently having illness were more likely to use a denture/bridge in the present study. Systemic ill health and functional limitations have been reported as 
Table 3 Multilevel prevalence ratios and $95 \%$ confidence intervals for denture/bridge use in males and females

\begin{tabular}{|c|c|c|c|c|c|c|c|c|}
\hline & \multicolumn{4}{|c|}{ Males } & \multicolumn{4}{|c|}{ Females } \\
\hline & \multicolumn{2}{|c|}{ Model 1} & \multicolumn{2}{|c|}{ Model 2} & \multicolumn{2}{|c|}{ Model 1} & \multicolumn{2}{|c|}{ Model 2} \\
\hline & PR & $95 \% \mathrm{Cl}$ & PR & $95 \% \mathrm{Cl}$ & PR & $95 \% \mathrm{Cl}$ & PR & $95 \% \mathrm{Cl}$ \\
\hline \multicolumn{9}{|l|}{ Fixed effects } \\
\hline \multicolumn{9}{|l|}{ Individual-level characteristics } \\
\hline \multicolumn{9}{|c|}{ Age group (years) (reference 65-69) } \\
\hline $70-74$ & 0.98 & $(0.94-1.02)$ & 0.98 & $(0.94-1.02)$ & 0.96 & $(0.92-1.00)^{\mathrm{a}}$ & 0.96 & $(0.92-1.00)$ \\
\hline $75-79$ & 0.96 & $(0.92-1.00)$ & 0.96 & $(0.92-1.00)$ & 0.97 & $(0.93-1.01)$ & 0.97 & $(0.93-1.02)$ \\
\hline $80-84$ & 0.98 & $(0.94-1.03)$ & 0.99 & $(0.94-1.04)$ & 0.98 & $(0.93-1.03)$ & 0.99 & $(0.94-1.04)$ \\
\hline$\geq 85$ & 0.99 & $(0.93-1.06)$ & 1.00 & $(0.93-1.06)$ & 0.99 & $(0.93-1.05)$ & 1.00 & $(0.94-1.06)$ \\
\hline \multicolumn{9}{|l|}{ Marital status (reference married) } \\
\hline Separated/divorced & 0.97 & $(0.93-1.02)$ & 0.97 & $(0.93-1.02)$ & 1.02 & $(0.99-1.06)$ & 1.02 & $(0.99-1.05)$ \\
\hline Never married & 0.91 & $(0.80-1.03)$ & 0.91 & $(0.81-1.03)$ & 0.98 & $(0.89-1.08)$ & 0.98 & $(0.89-1.08)$ \\
\hline Unknown/missing & 1.00 & $(0.89-1.11)$ & 1.00 & $(0.90-1.12)$ & 0.93 & $(0.84-1.02)$ & 0.93 & $(0.85-1.03)$ \\
\hline \multicolumn{9}{|c|}{ Educational attainment (years) (reference <6) } \\
\hline $6-9$ & 0.97 & $(0.86-1.10)$ & 0.97 & $(0.86-1.10)$ & 0.99 & $(0.92-1.08)$ & 0.99 & $(0.92-1.08)$ \\
\hline $10-12$ & 1.07 & $(0.95-1.21)$ & 1.07 & $(0.95-1.21)$ & 1.06 & $(0.98-1.15)$ & 1.06 & $(0.97-1.15)$ \\
\hline$\geq 13$ & 1.15 & $(1.02-1.30)^{\mathrm{a}}$ & 1.15 & $(1.01-1.30)^{a}$ & 1.07 & $(0.98-1.17)$ & 1.06 & $(0.97-1.16)$ \\
\hline Missing & 1.00 & $(0.87-1.15)$ & 1.00 & $(0.87-1.15)$ & 1.02 & $(0.92-1.13)$ & 1.02 & $(0.92-1.13)$ \\
\hline \multicolumn{9}{|c|}{ Equivalent income (10000 yen) (reference <50) } \\
\hline $50-99$ & 0.99 & $(0.89-1.10)$ & 0.99 & $(0.89-1.10)$ & 1.06 & $(0.99-1.15)$ & 1.06 & $(0.99-1.15)$ \\
\hline $100-149$ & 1.02 & $(0.92-1.13)$ & 1.02 & $(0.92-1.13)$ & 1.08 & $(1.00-1.17)^{\mathrm{a}}$ & 1.08 & $(1.00-1.16)$ \\
\hline $150-200$ & 1.10 & $(0.99-1.21)$ & 1.09 & $(0.99-1.21)$ & 1.11 & $(1.03-1.20)^{b}$ & 1.10 & $(1.03-1.19)^{b}$ \\
\hline $200-299$ & 1.14 & $(1.03-1.25)^{\mathrm{a}}$ & 1.13 & $(1.02-1.25)^{a}$ & 1.16 & $(1.08-1.24)^{\mathrm{c}}$ & 1.15 & $(1.07-1.23)^{c}$ \\
\hline $300-399$ & 1.18 & $(1.07-1.31)^{\mathrm{b}}$ & 1.18 & $(1.06-1.30)^{b}$ & 1.20 & $(1.12-1.30)^{c}$ & 1.19 & $(1.10-1.29)^{c}$ \\
\hline$\geq 400$ & 1.18 & $(1.06-1.31)^{b}$ & 1.17 & $(1.05-1.30)^{b}$ & 1.18 & $(1.09-1.27)^{c}$ & 1.17 & $(1.08-1.26)^{c}$ \\
\hline Missing & 1.04 & $(0.93-1.15)$ & 1.03 & $(0.93-1.15)$ & 1.03 & $(0.96-1.11)$ & 1.03 & $(0.96-1.11)$ \\
\hline \multicolumn{9}{|c|}{ Number of teeth (reference 10-19) } \\
\hline $1-9$ & 1.16 & $(1.13-1.20)^{c}$ & 1.16 & $(1.13-1.20)^{c}$ & 1.21 & $(1.17-1.25)^{c}$ & 1.21 & $(1.18-1.25)^{c}$ \\
\hline 0 & 1.05 & $(1.01-1.10)^{b}$ & 1.05 & $(1.01-1.10)^{a}$ & 1.12 & $(1.08-1.17)^{c}$ & 1.13 & $(1.08-1.17)^{c}$ \\
\hline \multicolumn{9}{|l|}{ Present illness (reference no) } \\
\hline Yes & 1.06 & $(1.02-1.10)^{b}$ & 0.94 & $(0.91-0.98)^{b}$ & 1.05 & $(1.01-1.09)^{b}$ & 0.95 & $(0.92-0.99)^{b}$ \\
\hline Missing & 1.05 & $(0.99-1.12)$ & 0.99 & $(0.94-1.05)$ & 1.08 & $(1.02-1.14)^{b}$ & 1.03 & $(0.98-1.08)$ \\
\hline \multicolumn{9}{|c|}{ Number of social groups (reference 0) } \\
\hline 1 & & & 1.02 & $(0.97-1.07)$ & & & 1.05 & $(1.01-1.10)^{a}$ \\
\hline 2 & & & 1.03 & $(0.98-1.09)$ & & & 1.05 & $(1.00-1.11)^{\mathrm{a}}$ \\
\hline $3-6$ & & & 1.05 & $(1.00-1.10)$ & & & 1.06 & $(1.01-1.11)^{\mathrm{a}}$ \\
\hline Missing & & & 1.02 & $(0.97-1.06)$ & & & 1.00 & $(0.97-1.04)$ \\
\hline \multicolumn{9}{|l|}{ Municipality-level characteristics } \\
\hline \multicolumn{9}{|l|}{ Number of dentists per 100000} \\
\hline \multicolumn{9}{|c|}{ people (reference lowest $(<47.29)$ ) } \\
\hline Low middle (47.29 - 53.97) & 1.03 & $(0.98-1.09)$ & 1.03 & $(0.98-1.09)$ & 1.04 & $(0.99-1.10)$ & 1.04 & $(0.99-1.10)$ \\
\hline High middle (53.98 - 59.74) & 0.99 & $(0.94-1.05)$ & 0.99 & $(0.94-1.05)$ & 0.97 & $(0.92-1.03)$ & 0.97 & $(0.92-1.03)$ \\
\hline Highest ( $\geq 59.75)$ & 0.98 & $(0.93-1.04)$ & 0.98 & $(0.93-1.04)$ & 0.95 & $(0.89-1.00)$ & 0.95 & $(0.89-1.00)$ \\
\hline
\end{tabular}


Table 3 Multilevel prevalence ratios and $95 \%$ confidence intervals for denture/bridge use in males and females (Continued)

\begin{tabular}{|c|c|c|c|c|c|c|c|c|}
\hline \multicolumn{9}{|c|}{ Population density (reference rural-agricultural) } \\
\hline Semi-urban & 1.07 & $(1.02-1.12)^{b}$ & 1.07 & $(1.02-1.13)^{b}$ & 1.10 & $(1.04-1.15)^{c}$ & 1.10 & $(1.04-1.15)^{\mathrm{C}}$ \\
\hline Urban & 1.10 & $(1.04-1.16)^{c}$ & 1.10 & $(1.05-1.16)^{c}$ & 1.11 & $(1.05-1.17)^{c}$ & 1.11 & $(1.05-1.17)^{\mathrm{C}}$ \\
\hline Metropolitan & 1.12 & $(1.06-1.18)^{c}$ & 1.13 & $(1.07-1.19)^{c}$ & 1.17 & $(1.10-1.24)^{c}$ & 1.17 & $(1.10-1.25)^{c}$ \\
\hline Intercept & 0.52 & $(0.44-0.60)^{c}$ & 0.54 & $(0.46-0.63)^{c}$ & 0.50 & $(0.45-0.56)^{c}$ & 0.51 & $(0.46-0.58)^{c}$ \\
\hline \multicolumn{9}{|l|}{ Random effects } \\
\hline Local district-level variance (SE) & 0.000 & 0.000 & 0.000 & 0.000 & 0.000 & 0.000 & 0.000 & 0.000 \\
\hline Municipality-level variance (SE) & 0.000 & 0.000 & 0.000 & 0.000 & 0.000 & 0.000 & 0.000 & 0.000 \\
\hline
\end{tabular}

barriers to seeking dental health care [25]. The results of the present study do not corroborate these findings. However, this discrepancy can be explained as follows. First, all subjects in the present study were ADL independent; therefore, functional limitations were not barriers to seeking dental health care in the present study. Second, subjects presently having illness may be more likely to ask dentists as well as doctors to solve their health problems, because a study using dental and medical care insurance records of employees aged 20-39 years showed that individuals who consulted dentists tended to receive medical treatment more frequently [26].

Although both sexes shared the same factors associated with denture/bridge use, there were differences in factors associated with denture/bridge use between sexes. The results of the present study showed that females involved in one or more kinds of social groups were more likely to use a denture/bridge. These results agree with an interview study from the UK that showed that patients focused on the social significance of oral rehabilitation when defining the need for a removable partial denture [16].

High educational attainment was associated with denture/bridge use only in males. Few studies have reported gender difference in the association between educational attainment and oral health status and/or oral health behavior, probably because most of the studies analyzed the association including both sexes [8-10,23,24]. A study in a Japanese older population showed that males with the highest educational attainment showed healthier ageing and lower mortality compared to males with the lowest educational attainment; however, no such differences were seen among females [27]. These results suggest that educational attainment is associated with oral and systemic health in males, but not in females. Further studies that confirm the reproducibility of these findings are needed to explain the gender difference.

No social capital variables were associated with denture/ bridge use. These results disagreed with those from a recent study that suggested that older people living in societies with rich social capital tend to have good oral health status, including having 20 or more teeth [18]. The results of the present study suggest that denture/bridge use was associated with personal factors, such as financial and social factors, but not social capital.

In contrast to social capital, high population density was associated with denture/bridge use in the present study. Because population density may be considered as surrogate information on socioeconomic status, it is possible that people living in richer areas tend to use denture/bridge.

The results of the present study show that target groups in which percentage of people using denture/bridge must be increased included people with low income and those living in the area with low population density. In addition, males with low educational attainment and females who do not have any social groups should be targeted. Taking this information into consideration, formulation of an intervention program for the target groups is recommended from the public health perspective.

The present study had a number of limitations. First, denture use was not distinguished from bridge use in the present study, which makes it difficult to interpret the results. A bridge is a fixed prosthesis and cannot be removed by patients; however, a denture can be removed by patients, and denture use is thus affected by patient compliance. To partially address this issue, we excluded subjects with 20 or more teeth and added number of teeth as a variable in the analyses.

Second, we did not obtain information regarding dental implants which is another type of dental prosthesis because dental implant is not covered by public health insurance in Japan. A recent national survey showed that $4.4 \%$, $1.2 \%$ and $2.8 \%$ of the whole population aged $65-74,75-84$ and 85- had dental implants, respectively [5]. Additional studies adding information on dental implants are necessary to confirm the results of the present study in the future. 
Third, the state of the denture such as stability and fit was unknown because this study was based on a self-administered questionnaire. Our previous study using a similar self-administered questionnaire showed that $13.7 \%$ of the participants with few teeth and dentures reported poorly fitted dentures [2]. Additional studies are needed to confirm the results of the present study using information on status of dentures.

Fourth, the measurements used were based on a selfadministered questionnaire. Some forms of bias, such as social desirability bias [28], may have affected the results of the present study. Fifth, because this was a crosssectional study, causal relationships are unclear.

\section{Conclusions}

Denture/bridge use was significantly associated with high economic status, present illness, and living in an area with high population density in both sexes among communitydwelling older Japanese having 19 or fewer teeth. Different factors were associated with denture/bridge use in males and females: high educational attainment in males and involvement in one or more social groups in females. Local district-level social capital was not associated with denture/bridge use.

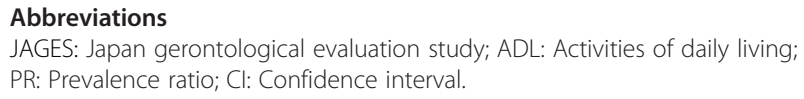

\section{Competing interests}

The authors declare that they have no competing interests.

\section{Authors' contributions}

TY conceived the idea for the study, participated in its design, performed the statistical analysis and drafted the manuscript as the principal author. KK is the principal investigator of the JAGES project, helped to develop the idea of the study, participated in acquiring the data and the study design, and edited the manuscript. JA, KS, JM, and MN participated in data acquisition and study design and critically revised the manuscript. SF helped with data analysis and critically revised the manuscript. YH helped to develop the idea of the study, participated in the study design, and edited the manuscript. All authors read and approved the final manuscript.

\section{Acknowledgements}

The present study used data from the Japan Gerontological Evaluation Study (JAGES). This study was supported in part by a grant of the Strategic Research Foundation Grant-aided Project for Private Universities from Ministry of Education, Culture, Sport, Science, and Technology, Japan (MEXT), 2009-2013 and Health Labour Sciences Research Grant, Comprehensive Research on Aging and Health (H24-Junkanki(Seisyu)-Ippan-007) from the Japanese Ministry of Health, Labour and Welfare (MHLW).

\section{Author details}

${ }^{1}$ Department of Dental Sociology, Kanagawa Dental University Graduate School of Dentistry, 82 Inaoka-cho, Yokosuka, Kanagawa 238-8580, Japan. ${ }^{2}$ Center for Preventive Medical Science, Chiba University, Chiba, Japan. ${ }^{3}$ Center for Well-being and Society, Nihon Fukushi University, Nagoya, Japan. ${ }^{4}$ Department of International and Community Oral Health, Tohoku University Graduate School of Dentistry, Sendai, Japan. ${ }^{5}$ Department of Policy Studies, Aichi Gakuin University, Nisshin, Japan. ${ }^{6}$ Faculty of Sociology, Rikkyo University, Tokyo, Japan. ${ }^{7}$ Department of Nutrition, Faculty of Health and Nutrition, Tokaigakuen University, Nagoya, Japan.
Received: 29 January 2014 Accepted: 28 May 2014

Published: 3 June 2014

\section{References}

1. Kanehisa Y, Yoshida M, Taji T, Akagawa Y, Nakamura H: Body weight and serum albumin change after prosthodontic treatment among institutionalized elderly in a long-term care geriatric hospital. Community Dent Oral Epidemiol 2009, 37:534-538.

2. Yamamoto T, Kondo K, Hirai H, Nakade M, Aida J, Hirata Y: Association between self-reported dental health status and onset of dementia: a 4-year prospective cohort study of older Japanese adults from the Aichi Gerontological Evaluation Study (AGES) Project. Psychosom Med 2012, 74:241-248

3. Yamamoto T, Kondo K, Misawa J, Hirai H, Nakade M, Aida J, Kondo N, Kawachi I, Hirata Y: Dental status and incident falls among older Japanese: a prospective cohort study. BMJ Open 2012, 2:e001262.

4. Yoshida M, Morikawa H, Yoshikawa M, Tsuga K, Akagawa Y: Eight-year mortality associated with dental occlusion and denture use in community-dwelling elderly persons. Gerodontology 2005, 22:234-237.

5. Ministry of Health, Labour and Welfare, Japan: The Survey of Dental Diseases (2011). http://www.mhlw.go.jp/toukei/list/62-23.html (in Japanese).

6. Jepson NJ, Thomason JM, Steele JG: The influence of denture design on patient acceptance of partial dentures. Br Dent J 1995, 178:296-300.

7. Schou L: Oral health, oral health care, and oral health promotion among older adults: social and behavioral dimensions. In Disease Prevention and Oral Health Promotion. Socio-Dental Science in Action. Edited by Cohen LK, Gift HC. Copenhagen: Munksgaard; 1995:213-270.

8. Österberg T, Lundgren M, Emilson CG, Sundh V, Birkhed D, Steen B: Utilization of dental services in relation to socioeconomic and health factors in the middle-aged and elderly Swedish population. Acta Odontol Scand 1998, 56:41-47.

9. Lupi-Pegurier L, Clerc-Urmes I, Abu-Zaineh M, Paraponaris A, Ventelou B: Density of dental practitioners and access to dental care for the elderly: a multilevel analysis with a view on socio-economic inequality. Health Policy 2011, 103:160-167.

10. Phipps KR, Reifel N, Bothwell E: The oral health status, treatment needs, and dental utilization patterns of Native American elders. J Public Health Dent 1991, 51:228-233.

11. Merelie DL, Heyman B: Dental needs of the elderly in residential care in Newcastle-upon-Tyne and the role of formal carers. Community Dent Oral Epidemiol 1992, 20:106-111.

12. Hanibuchi T, Aida J, Nakade M, Hirai H, Kondo K: Geographical accessibility to dental care in the Japanese elderly. Community Dent Health 2011, 28:128-135.

13. Kiyak HA, Miller RR: Age differences in oral health attitudes and dental service utilization. J Public Health Dent 1982, 42:29-41.

14. Antczak AA, Branch LG: Perceived barriers to the use of dental services by the elderly. Gerodontics 1985, 1:194-198.

15. Matsuyama Y, Aida J, Takeuchi K, Tsakos G, Watt RG, Kondo K, Osaka K: Inequalities of dental prosthesis use under universal health care insurance. Community Dent Oral Epidemiol 2014, 42:122-128.

16. Graham R, Mihaylov S, Jepson N, Allen PF, Bond S: Determining "need" for a removable partial denture: a qualitative study of factors that influence dentist provision and patient use. Br Dent J 2006, 200:155-158.

17. Derose KP, Varda DM: Social capital and health care access: a systematic review. Med Care Res Rev 2009, 66:272-306.

18. Aida J, Hanibuchi T, Nakade M, Hirai H, Osaka K, Kondo K: The different effects of vertical social capital and horizontal social capital on dental status: a multilevel analysis. Soc Sci Med 2009, 69:512-518.

19. Takeuchi K, Aida J, Kondo K, Osaka K: Social participation and dental health status among older Japanese adults: a population-based cross-sectional study. PLoS One 2013, 8:e61741.

20. Yamamoto T, Kondo K, Fuchida S, Aida J, Nakade M, Hirata Y: Validity of self-reported oral health variables: Aichi Gerontological Evaluation Study (AGES) project. Health Sci Health Care 2012, 12:4-12.

21. Zhang J, Yu KF: What's the relative risk? A method of correcting the odds ratio in cohort studies of common outcomes. JAMA 1998, 280:1690-1691.

22. McNutt LA, Wu C, Xue $X$, Hafner JP: Estimating the relative risk in cohort studies and clinical trials of common outcomes. Am J Epidemiol 2003, 157:940-943. 
23. Donaldson AN, Everitt B, Newton T, Steele J, Sherriff M, Bower E: The effects of social class and dental attendance on oral health. J Dent Res 2008, 87:60-64.

24. Locker D, Maggirias J, Quinonez C: Income, dental insurance coverage, and financial barriers to dental care among Canadian adults. J Public Health Dent 2011, 71:327-334.

25. Jones JA, Adelson R, Niessen LC, Gilbert GH: Issues in financing dental care for the elderly. J Public Health Dent 1990, 50:268-275.

26. Yamamoto T, Sugano A, Morita M, Tada T, Watanabe T: Workers who visit dentists consult medical doctors more frequently. J Dent Health 2003, 53:531-534 (in Japanese).

27. Kondo K, Ashida T, Hirai H, Misawa J, Suzuki K: The relationship between socio-economic status and the loss of healthy aging, and relevant gender differences in the Japanese older population: AGES Project longitudinal study. Iryo To Syakai 2012, 22:19-30 (in Japanese).

28. Sanzone LA, Lee JY, Divaris K, DeWalt DA, Baker AD, Vann WF Jr: A cross sectional study examining social desirability bias in caregiver reporting of children's oral health behaviors. BMC Oral Health 2013, 13:24.

doi:10.1186/1472-6831-14-63

Cite this article as: Yamamoto et al:: Social determinants of denture/ bridge use: Japan gerontological evaluation study project cross-sectional study in older Japanese. BMC Oral Health 2014 14:63.

\section{Submit your next manuscript to BioMed Central and take full advantage of:}

- Convenient online submission

- Thorough peer review

- No space constraints or color figure charges

- Immediate publication on acceptance

- Inclusion in PubMed, CAS, Scopus and Google Scholar

- Research which is freely available for redistribution 